\title{
Folding Theory Applied to Residuated Lattices
}

\author{
Albert Kadji, ${ }^{1}$ Celestin Lele, ${ }^{2}$ Jean B. Nganou, ${ }^{3}$ and Marcel Tonga ${ }^{1}$ \\ ${ }^{1}$ Departmen of Mathematics, University of Yaounde 1, P.O. Box 812, Yaounde, Cameroon \\ ${ }^{2}$ Departmen of Mathematics, University of Dschang, P.O. Box 67, Dschang, Cameroon \\ ${ }^{3}$ Departmen of Mathematics, University of Oregon, Eugene, OR 97403, USA \\ Correspondence should be addressed to Celestin Lele; celestinlele@yahoo.com \\ Received 14 February 2014; Revised 20 April 2014; Accepted 20 May 2014; Published 25 June 2014 \\ Academic Editor: Radko Mesiar \\ Copyright (C) 2014 Albert Kadji et al. This is an open access article distributed under the Creative Commons Attribution License, \\ which permits unrestricted use, distribution, and reproduction in any medium, provided the original work is properly cited. \\ Residuated lattices play an important role in the study of fuzzy logic based on $t$-norms. In this paper, we introduce some notions of \\ $n$-fold filters in residuated lattices, study the relations among them, and compare them with prime, maximal and primary, filters. This \\ work generalizes existing results in BL-algebras and residuated lattices, most notably the works of Lele et al., Motamed et al., Haveski \\ et al., Borzooei et al., Van Gasse et al., Kondo et al., Turunen et al., and Borumand Saeid et al., we draw diagrams summarizing the \\ relations between different types of $n$-fold filters and $n$-fold residuated lattices.
}

\section{Introduction}

Since Hájek introduced his basic fuzzy logics, (BL-logics in short) in 1998 [1], as logics of continuous $t$-norms, a multitude of research papers related to the algebraic counterparts of BLlogics, has been published. In [2-5], the authors defined the notions of $n$-fold (implicative, positive implicative, Boolean, fantastic, obstinate, and normal) filters in BL-algebras and studied the relation among them.

A close analysis of the situation reveals that the main drive in all the previously mentioned works resides in the existence of an adjoint pair of operations. Just as the foldness theory for filters in BL-algebras generalizes filters introduced by Hájek, our foldness theory for filters in residuated lattices builds on recently published works on filters in residuated lattices by Haveshki et al. [6], Van Gasse et al. [7], Kondo and Dudek [8], Kondo and Turunen [9], Borumand Saeid and Pourkhatoun in [10], and Zahiri and Farahani in [11].

More specifically, we introduce the notions of $n$-fold (implicative, positive implicative, Boolean, fantastic, normal, integral, and involutive) filters and $n$-fold Boolean filters of the second kind in residuated lattices, notions that naturally generalize the corresponding ones previously studied in BL-algebras. Concurrently, we introduce the same foldness concepts on residuated lattices. In each folding class, we tie together the two concepts by characterizing the corresponding residuated lattices using their filters. For instance, it is shown (Proposition 20) that a residuated lattice is $n$-fold implicative if and only if its trivial filter is $n$-fold implicative if and only if all its filters are $n$-fold implicative. Examples are included not only to illustrate the newly introduced concepts but also to differentiate them from the existing ones. Finally, diagrams summarizing all the relationships between the above classes of filters and residuated lattices are given (see Figures 1 and 2) for quick referencing. It should be noted that when restricted to BL-algebras these diagrams contain previously discovered relationships and also some newly found ones.

\section{Preliminaries}

A residuated lattice is a nonempty set $L$ with four binary operations $\wedge, \vee, \otimes, \rightarrow$, and two constants 0,1 satisfying the following properties:

$\mathrm{L}-1:(L, \wedge, \vee, 0,1)$ is a bounded lattice.

$\mathrm{L}-2:(L, \otimes, 1)$ is a commutative monoid.

L-3: $x \otimes y \leq z$ if and only if $x \leq y \rightarrow z$ (residuation). 


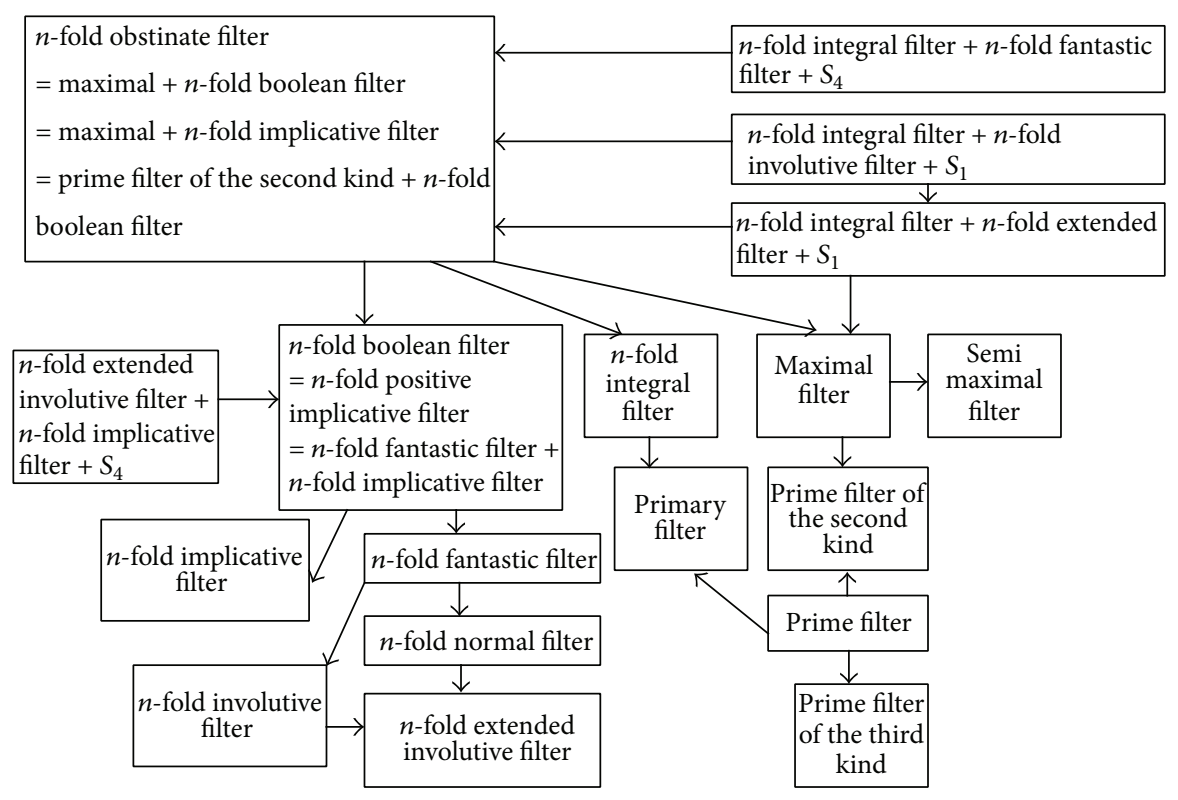

FIGURE 1: Diagram of $n$-fold filters.

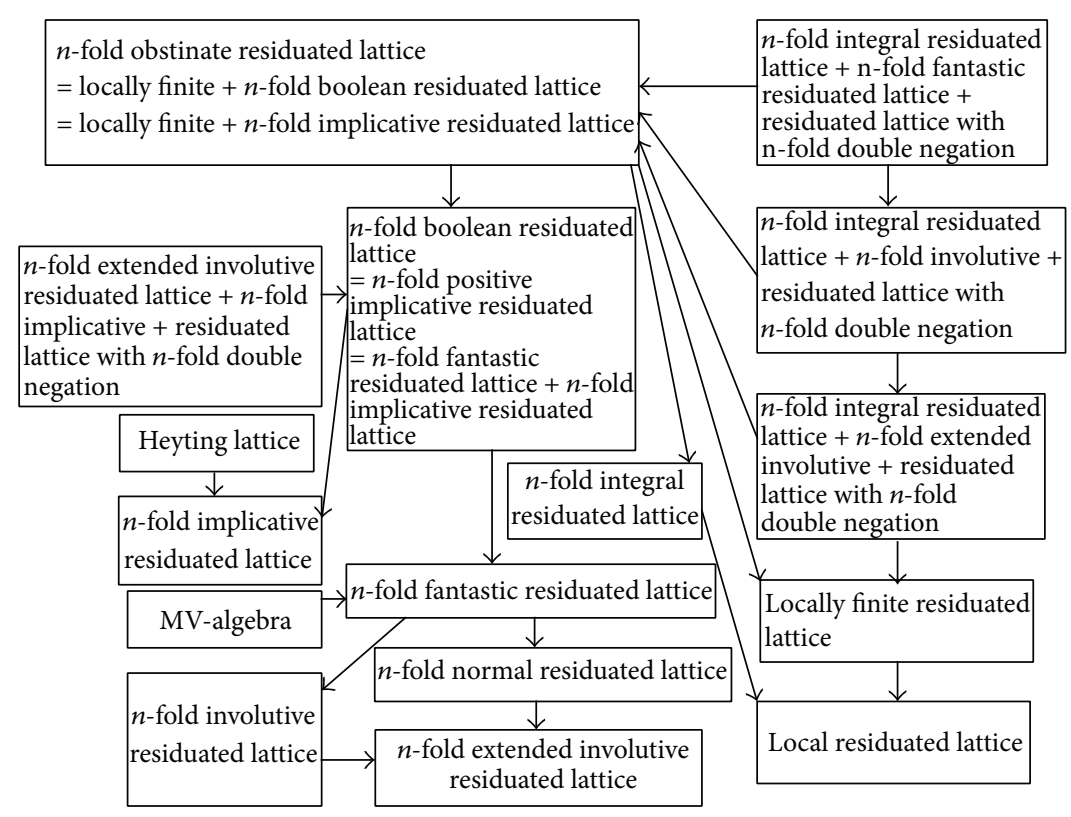

FIGURE 2: Diagrams of $n$-fold residuated lattices.

A MTL-algebra is a residuated lattice $L$ which satisfies the following condition:

$$
\text { L-4: }(x \rightarrow y) \vee(y \rightarrow x)=1 \text { (prelinearity) }
$$

A BL-algebra is a MTL-algebra $L$ which satisfies the following condition:

$$
\text { L-5: } x \wedge y=x \otimes(x \rightarrow y) \text { (divisibility). }
$$

A $M V$-algebra is a BL-algebra $L$ which satisfies the following condition:

L-6: $\overline{\bar{x}}=x$, where $\bar{x}:=x \rightarrow 0$. Alternatively, a $M V$ algebra can be defined as a residuated lattice $L$ which satisfies the following condition:

L-7: $(x \rightarrow y) \rightarrow y=(y \rightarrow x) \rightarrow x$.

A Heyting lattice is a residuated lattice $L$ which satisfies the following condition:

L-8: $x \wedge y=x \otimes y$. 
A Boolean lattice is a residuated lattice $L$ which satisfies the following condition:

$$
\text { L-9: } x \vee \bar{x}=1 \text {. }
$$

In this work, unless mentioned otherwise, $(L, \wedge, \vee, \otimes, \rightarrow$, $0,1)$ will be a residuated lattice, which will often be referred to by its support set $L$.

For any element $x \in L$, we define $x^{0}=1$ and $x^{n+1}=$ $x^{n} \otimes x$ for any integer $n \geq 0$.

Proposition 1 (see $[7,9,10,12])$. The following properties hold in a residuated lattice.

(1) $x \leq y$ if and only if $x \rightarrow y=1 ; x \otimes y \leq x \wedge y$.

(2) $x \rightarrow(y \rightarrow z)=(x \otimes y) \rightarrow z$.

(3) $x \rightarrow(y \rightarrow z)=y \rightarrow(x \rightarrow z)$.

(4) If $x \leq y$, then $y \rightarrow z \leq x \rightarrow z$ and $z \rightarrow x \leq z \rightarrow$ $y$.

(5) $x \leq y \rightarrow(x \otimes y) ; x \otimes(x \rightarrow y) \leq y$.

(6) $y \leq(x \rightarrow y) ; x \leq \overline{\bar{x}}$, and $\overline{\bar{x}}=\bar{x}$.

(7) $x \otimes y=0$ if and only if $x \leq \bar{y}$.

(8) If $x \leq y$, then $x \otimes z \leq y \otimes z$ and $\bar{y} \leq \bar{x}$.

(9) $\overline{x \otimes y}=x \rightarrow \bar{y}$.

(10) $x \vee y=1$ implies $x \otimes y=x \wedge y$ and $x^{n} \vee y^{n}=$ 1 for every $n \geq 1$.

(11) $x \otimes(y \vee z)=(x \otimes y) \vee(x \otimes z)$.

(12) $(x \vee y) \rightarrow z=(x \rightarrow z) \wedge(y \rightarrow z) ;(x \rightarrow z) \vee(y \rightarrow$ $z) \leq(x \wedge y) \rightarrow z$.

(13) $x \vee y \leq((x \rightarrow y) \rightarrow y) \wedge((y \rightarrow x) \rightarrow x)$.

(14) $x \rightarrow y \leq(y \rightarrow z) \rightarrow(x \rightarrow z)$.

(15) $x \rightarrow y \leq(z \rightarrow x) \rightarrow(z \rightarrow y)$.

Let $(L, \wedge, \vee, \otimes, \rightarrow, 0,1)$ and $F$ be a nonempty subset of $L$. Then, $F$ is called a filter if it satisfies the following two conditions.

(F1): for every $x, y \in F, x \otimes y \in F$.

(F2): for every $x, y \in L$, if $x \leq y$ and $x \in F$, then $y \in F$.

$F$ is called a deductive system if $1 \in F$, and for all $x, y \in L$, $x \rightarrow y \in F$ and $x \in F$ implies $y \in F$.

It is known that in a residuated lattice, filters and deductive systems coincide [7].

The residuated lattices listed below are not BL-algebras and will be used to illustrate the concepts treated in the paper.
Example 2. Let $L=\{0, a, b, c, d, 1\}$ be a lattice such that $0<$ $a<c, 0<b<c<d<1$, where $a$ and $b$ are not comparable. Define the operations $\otimes$ and $\rightarrow$ by the two tables shown in (1). Then $L$ is a residuated lattice which is not a MTL-algebra since $(a \rightarrow b) \vee(b \rightarrow a)=c \neq 1$ :

\begin{tabular}{l|l|l|l|l|l|l}
$\otimes$ & 0 & $a$ & $b$ & $c$ & $d$ & 1 \\
\hline 0 & 0 & 0 & 0 & 0 & 0 & 0 \\
$a$ & 0 & $a$ & 0 & $a$ & $a$ & $a$ \\
$b$ & 0 & 0 & $b$ & $b$ & $b$ & $b$ \\
$c$ & 0 & $a$ & $b$ & $c$ & $c$ & $c$ \\
$d$ & 0 & $a$ & $b$ & $c$ & $c$ & $d$ \\
1 & 0 & $a$ & $b$ & $c$ & $d$ & 1
\end{tabular}

\begin{tabular}{c|c|c|c|c|c|c}
$\longrightarrow$ & 0 & $a$ & $b$ & $c$ & $d$ & 1 \\
\hline 0 & 1 & 1 & 1 & 1 & 1 & 1 \\
$a$ & $b$ & 1 & $b$ & 1 & 1 & 1 \\
$b$ & $a$ & $a$ & 1 & 1 & 1 & 1 \\
$c$ & 0 & $a$ & $b$ & 1 & 1 & 1 \\
$d$ & 0 & $a$ & $b$ & $d$ & 1 & 1 \\
1 & 0 & $a$ & $b$ & $c$ & $d$ & 1
\end{tabular}

$F=\{1, b, c, d\} ; F_{1}=\{1, a, c, d\} ; F_{2}=\{1, c, d\}$ are the proper nontrivial filters of $L$.

Example 3. Let $L=\{0, a, b, c, d, 1\}$ be a lattice such that $0<$ $a<c<d<1$ and $0<b<c<d<1$, where $a$ and $b$ are not comparable. Define the operations $\otimes$ and $\rightarrow$ by the two tables shown in (2). Then $(L, \wedge, \vee, \otimes, \rightarrow, 0,1)$ is a residuated lattice which is not a MTL-algebra, since $(a \rightarrow b) \vee(b \rightarrow$ a) $=c \vee c=c \neq 1$ :

\begin{tabular}{l|l|l|l|l|l|l}
$\otimes$ & 0 & $a$ & $b$ & $c$ & $d$ & 1 \\
\hline 0 & 0 & 0 & 0 & 0 & 0 & 0 \\
$a$ & 0 & 0 & 0 & 0 & $a$ & $a$ \\
$b$ & 0 & 0 & 0 & 0 & $b$ & $b$ \\
$c$ & 0 & 0 & 0 & 0 & $c$ & $c$ \\
$d$ & 0 & $a$ & $b$ & $c$ & $d$ & $d$ \\
1 & 0 & $a$ & $b$ & $c$ & $d$ & 1 \\
$\longrightarrow$ & 0 & $a$ & $b$ & $c$ & $d$ & 1 \\
\hline 0 & 1 & 1 & 1 & 1 & 1 & 1 \\
$a$ & $c$ & 1 & $c$ & 1 & 1 & 1 \\
$b$ & $c$ & $c$ & 1 & 1 & 1 & 1 \\
$c$ & $c$ & $c$ & $c$ & 1 & 1 & 1 \\
$d$ & 0 & $a$ & $b$ & $c$ & 1 & 1 \\
1 & 0 & $a$ & $b$ & $c$ & $d$ & 1
\end{tabular}

$F=\{1, d\}$ is the only nontrivial filter of $L$.

Example 4. Let $L=\{0, a, b, c, 1\}$ be a lattice such that $0<a<$ $b<c<1$. Define the operations $\otimes$ and $\rightarrow$ by the two tables 
shown in (3). Then $(L, \wedge, \vee, \otimes, \rightarrow, 0,1)$ is a residuated lattice which is not a BL-algebra, since $c \otimes(c \rightarrow b)=a \neq b=c \wedge b$ :

\begin{tabular}{l|l|l|l|l|l}
$\otimes$ & 0 & $a$ & $b$ & $c$ & 1 \\
\hline 0 & 0 & 0 & 0 & 0 & 0 \\
$a$ & 0 & $a$ & $a$ & $a$ & $a$ \\
$b$ & 0 & $a$ & $a$ & $a$ & $b$ \\
$c$ & 0 & $a$ & $a$ & $c$ & $c$ \\
1 & 0 & $a$ & $b$ & $c$ & 1 \\
$\longrightarrow$ & 0 & $a$ & $b$ & $c$ & 1 \\
\hline 0 & 1 & 1 & 1 & 1 & 1 \\
$a$ & 0 & 1 & 1 & 1 & 1 \\
$b$ & 0 & $c$ & 1 & 1 & 1 \\
$c$ & 0 & $b$ & $b$ & 1 & 1 \\
1 & 0 & $a$ & $b$ & $c$ & 1
\end{tabular}

$F_{1}=\{1\}, F_{2}=\{1, c\}, F_{3}=\{1, a, b, c\}$ are filters of $L$.

Example 5. Let $L=\{0, a, b, c, 1\}$ be a lattice such that $0<$ $a<c<1,0<b<c<1$, where $a$ and $b$ are not comparable. Define the operations $\otimes$ and $\rightarrow$ by the two tables shown in (4). Then $(L, \wedge, \vee, \otimes, \rightarrow, 0,1)$ is a residuated lattice which is not a BL-algebra, since $(a \rightarrow b) \vee(b \rightarrow a)=a \vee b=c \neq 1$ :

\begin{tabular}{l|l|l|l|l|l}
$\otimes$ & 0 & $a$ & $b$ & $c$ & 1 \\
\hline 0 & 0 & 0 & 0 & 0 & 0 \\
$a$ & 0 & $a$ & 0 & $a$ & $a$ \\
$b$ & 0 & 0 & $b$ & $b$ & $b$ \\
$c$ & 0 & $a$ & $b$ & $c$ & $c$ \\
1 & 0 & $a$ & $b$ & $c$ & 1 \\
$\longrightarrow$ & 0 & $a$ & $b$ & $c$ & 1 \\
\hline 0 & 1 & 1 & 1 & 1 & 1 \\
$a$ & $b$ & 1 & $b$ & 1 & 1 \\
$b$ & $a$ & $a$ & 1 & 1 & 1 \\
$c$ & 0 & $a$ & $b$ & 1 & 1 \\
1 & 0 & $a$ & $b$ & $c$ & 1
\end{tabular}

$F_{1}=\{1\}, F_{2}=\{1, c\}, F_{3}=\{1, a, c\}, F_{4}=\{1, b, c\}$ are filters of $L$.

Example 6. Let $L=\{0, a, b, c, 1\}$ be a lattice such that $0<a<$ $c<1,0<b<c<1$, where $a$ and $b$ are not comparable. Define the operations $\otimes$ and $\rightarrow$ by the two tables shown in (5). Then $(L, \wedge, \vee, \otimes, \rightarrow, 0,1)$ is a residuated lattice which is not a MTL-algebra, since $(a \rightarrow b) \vee(b \rightarrow a)=c \vee c=c \neq 1$ :

$$
\begin{array}{l|l|l|l|l|l}
\otimes & 0 & a & b & c & 1 \\
\hline 0 & 0 & 0 & 0 & 0 & 0 \\
a & 0 & 0 & 0 & 0 & a \\
b & 0 & 0 & 0 & 0 & b \\
c & 0 & 0 & 0 & 0 & c \\
1 & 0 & a & b & c & 1 \\
\longrightarrow & 0 & a & b & c & 1 \\
\hline 0 & 1 & 1 & 1 & 1 & 1 \\
a & c & 1 & c & 1 & 1 \\
b & c & c & 1 & 1 & 1 \\
c & c & c & c & 1 & 1 \\
1 & 0 & a & b & c & 1
\end{array}
$$

$F=\{1\}$ is the only proper filter of $L$.
Remark 7 (see [13]). It is well known that the class of residuated lattices is a variety. So from the above examples, we may obtain infinite residuated lattices which are not $M T L$ algebras.

Definition 8 (see [14]). Let $L$ be a residuated lattice.

(i) $L$ is said to be locally finite if for every $x \neq 1$, there exists an integer $n \geq 1$ such that $x^{n}=0$.

(ii) $L$ is said to be local if it has a unique maximal filter.

Clearly, a locally finite residuated lattice is local.

Given a filter $F$ of a residuated lattice $L$, there is a wellknown congruence $\equiv_{F}$ on $L$ defined by $x \equiv_{F} y$ if and only if $(x \rightarrow y \in F$ and $y \rightarrow x \in F)$; the quotient structure $L / F$ is also a residuated lattice where $x / F \wedge y / F=(x \wedge y) / F ; x / F \vee$ $y / F=(x \vee y) / F ; x / F \otimes y / F=(x \otimes y) / F ; x / F \rightarrow y / F=(x \rightarrow$ $y) / F$.

One can easily verify the following result.

Proposition 9 (see [14]). For any filter $F$ of a residuated lattices $L$, the following conditions are equivalent.

(i) $F$ is a maximal filter of $L$.

(ii) For any $x \in L, x \notin F$ if and only if $\overline{x^{m}} \in F$ for some $m \geq 1$.

(iii) $L / F$ is a locally finite residuated lattice.

Consequently, it is straightforward to see that $L$ is locally finite if and only if $\{1\}$ is a maximal filter, and $L$ is local if and only if $D(L):=\left\{x \in L ; x^{m} \neq 0 \forall m \geq 1\right\}$ is a proper filter.

Definition 10. Let $L_{1}$ and $L_{2}$ be two residuated lattices. Then a map $f: L_{1} \rightarrow L_{2}$ is called a residuated lattice homomorphism if it satisfies the following conditions.

(i) $f(0)=0$.

(ii) $f(x \otimes y)=f(x) \otimes f(y)$, for every $x, y \in L_{1}$.

(iii) $f(x \rightarrow y)=f(x) \rightarrow f(y)$, for every $x, y \in L_{1}$.

If $f$ is bijective, the homomorphism $f$ is called a residuated lattice isomorphism. In this case we write $L_{1} \cong L_{2}$.

Definition 11 (see $[7,9,14,15])$. A proper filter $F$ is said to be:

(i) prime if, for all $x, y \in L, x \rightarrow y \in F$ or $y \rightarrow x \in F$;

(ii) prime of the second kind if, for all $x, y \in L, x \vee y \in F$ implies $x \in F$ or $y \in F$;

(iii) prime of the third kind if, for all $x, y \in L,(x \rightarrow y) \vee$ $(y \rightarrow x) \in F$

(iv) boolean if, for all $x \in L, x \vee \bar{x} \in F$;

(v) boolean of the second kind if, for all $x \in L, x \in F$ or $\bar{x} \in F$;

(vi) primary if, for all $x, y \in L: \overline{(x \otimes y)} \in F$ implies $\overline{x^{n}} \in F$ or $\overline{y^{n}} \in F$, for some integer $n \geq 0$;

(vii) a semimaximal filter if $\operatorname{Rad}(F)=F$, where $\operatorname{Rad}(F)$ is the intersection of all maximal filters of $L$ which contain $F$. 
Remark 12 (see $[7,9,14,15]$ ). (i) Prime filters are prime filters of the second kind. The converse is true if $L$ is a MTL-algebra.

(ii) Prime filters are prime filters of the third kind.

(iii) Boolean filters of the second kind are boolean filters. filters.

(iv) If $L$ is a MTL-algebra, then maximal filters are prime

(v) If a filter is prime of the second kind and boolean, then it is boolean of the second kind.

(vi) Maximal filters are semi maximal filters.

Lemma 13. Maximal filters are prime filters of the second kind.

Proof. Assume that $P$ is a maximal filter of $L$ and let $P_{1}, P_{2}$ be two proper filters of $L$ such that $P_{1} \cap P_{2}=P$. Then $P \subseteq P_{1}$ and $P \subseteq P_{2}$; hence $P=P_{1}$ and $P=P_{2}$ since $P$ is a maximal filter and $P_{1}, P_{2}$ proper filters. From [14, Lemma 3], it follows that $P$ is prime of the second kind.

Proposition 14. Any prime filter of $L$ is a primary filter of $L$.

Proof. Using Definition 11(vi), the proof is as shown in the case of pseudo-BL algebra [16].

Now, unless mentioned otherwise, $n \geq 1$ will be an integer and $F \subseteq L$.

A class $\mathscr{F}$ of filters of $L$ will be said to be closed under extension if, for any filters $F_{1}$ and $F_{2}$ of $L, F_{1} \in \mathscr{F}$ and $F_{1} \subseteq F_{2}$ imply $F_{2} \in \mathscr{F}$.

\section{3. $n$-Fold Implicative Filter}

Definition 15. Let $L$ be a residuated lattice.

(i) $L$ is said to be $n$-fold implicative if $x^{n+1}=x^{n}$ for all $x \in L$.

(ii) A subset $F \subseteq L$ is called an $n$-fold implicative filter if $1 \in F$ and for all $x, y, z \in L, x^{n} \rightarrow(y \rightarrow z) \in F$ and $x^{n} \rightarrow y \in F$ imply $x^{n} \rightarrow z \in F$.

In particular, a 1-fold implicative filter is an implicative filter [12].

By taking $x=1$ in the definition, we see that any $n$-fold implicative filter is a filter.

Like in the case of $B L$-algebras [8], a 1-fold implicative residuated lattice may be called a Gödel residuated lattice.

Example 16. Let $n \geq 1$. The residuated lattice of Example 5 is $n$-fold implicative. But the residuated lattice of Example 6 is not 1-fold implicative, since $a^{2}=0 \neq a=a^{1}$.

Lemma 17. A filter $F$ of $L$ is $n$-fold implicative if and only if, for all $a \in L, F_{a}:=\left\{b \in L ; a^{n} \rightarrow b \in F\right\}$ is a filter of $L$.

Proof. $(\Rightarrow)$ : Let $F$ be an $n$-fold implicative filter of $L$. Since $a^{n} \rightarrow 1=1 \in F$, we have $1 \in F_{a}$. Let $x, y \in L$ be such that $x, x \rightarrow y \in F_{a}$; then $a^{n} \rightarrow x \in F$ and $a^{n} \rightarrow(x \rightarrow y) \in F$. So, $a^{n} \rightarrow y \in F$, and $y \in F_{a}$, showing that $F_{a}$ is a filter of $L$.

$(\Leftarrow)$ : Conversely, suppose that $F_{a}$ is a filter of $L$, for all $a \in L$. Let $x, y, z \in L$ such that $x^{n} \rightarrow(y \rightarrow z) \in F$ and $x^{n} \rightarrow y \in F$. Then $y, y \rightarrow z \in F_{x}$, and thus $z \in F_{x}$, so, $x^{n} \rightarrow z \in F$.

Proposition 18. The following conditions are equivalent for a filter $F$ and $n \geq 1$.

(i) $F$ is $n$-fold implicative.

(ii) For all $x \in L, x^{n} \rightarrow x^{2 n} \in F$.

(iii) For all $x, y \in L, x^{n+1} \rightarrow y \in F$ imply $x^{n} \rightarrow y \in F$.

Moreover, the class of $n$-fold implicative filters is closed under extension.

Proof. (i) $\Rightarrow$ (ii): Let $x \in L$; by Proposition 1(2), we have $x^{n} \rightarrow\left(x^{n} \rightarrow x^{2 n}\right)=x^{2 n} \rightarrow x^{2 n}=1 \in F$ and $x^{n} \rightarrow x^{n}=1 \in F$. Since $F$ is an $n$-fold implicative filter of $L$, we have $x^{n} \rightarrow x^{2 n} \in F$.

(ii) $\Rightarrow$ (i): Let $x, y, z \in L$ such that $x^{n} \rightarrow(y \rightarrow z) \in F$ and $x^{n} \rightarrow y \in F$. By Proposition 1(5) we have $x^{2 n} \otimes\left(x^{n} \rightarrow\right.$ $y) \otimes\left[x^{n} \rightarrow(y \rightarrow z)\right] \leq z$, so $\left(\left[x^{n} \rightarrow(y \rightarrow z)\right] \otimes\left(x^{n} \rightarrow\right.\right.$ $y)) \leq x^{2 n} \rightarrow z$. (*)

Since $\left[x^{n} \rightarrow(y \rightarrow z)\right] \otimes\left(x^{n} \rightarrow y\right) \in F$, we obtain that $x^{2 n} \rightarrow z \in F$. Now, by Proposition 1(14), $x^{n} \rightarrow x^{2 n} \leq$ $\left(x^{2 n} \rightarrow z\right) \rightarrow\left(x^{n} \rightarrow z\right)$. So, by the hypothesis and $(*)$, we obtain $x^{n} \rightarrow z \in F$.

(i) $\Rightarrow$ (iii): Let $x, y \in L$ be such that $x^{n+1} \rightarrow y \in F$. By Proposition 1(2), we have $x^{n} \rightarrow(x \rightarrow y)=x^{n+1} \rightarrow y \in F$ and $x^{n} \rightarrow x=1 \in F$; since $F$ is an $n$-fold implicative filter of $L$ we have $x^{n} \rightarrow y \in F$.

(iii) $\Rightarrow$ (ii):

Firstly, for $n=1, x^{1+1} \rightarrow x^{2}=1 \in F$; from (iii), we have $x \rightarrow x^{2} \in F$.

Secondly, for $n=2, x^{2+1} \rightarrow\left(x^{2-1} \rightarrow x^{4}\right)=x^{4} \rightarrow$ $x^{4} \in F$; from (iii) we have $x^{2} \rightarrow\left(x \rightarrow x^{4}\right) \in F$; that is $x^{3} \rightarrow x^{4} \in F$. From (iii) again, we obtain $x^{2} \rightarrow$ $x^{4} \in F$.

Finally for $n>2$, note that $x^{n+1} \rightarrow\left(x^{n-1} \rightarrow x^{2 n}\right)=$ $x^{2 n} \rightarrow x^{2 n}=1 \in F$. From (iii) we obtain $x^{n} \rightarrow$ $\left(x^{n-1} \rightarrow x^{2 n}\right) \in F$; that is $x^{n+1} \rightarrow\left(x^{n-2} \rightarrow x^{2 n}\right) \epsilon$ $F$. From (iii) again, we also have $x^{n} \rightarrow\left(x^{n-2} \rightarrow\right.$ $\left.x^{2 n}\right) \in F$.

By repeating the process $n$ times, we obtain $x^{n} \rightarrow$ $\left(x^{n-n} \rightarrow x^{2 n}\right)=x^{n} \rightarrow x^{2 n} \in F$.

Using Propositions 18 and 1, we can easily see that an $n$-fold implicative filter is $(n+1)$-fold implicative. But the converse is not true, as illustrated by Example 6, where the filter $\{1\}$ is 2 -fold implicative but not 1-fold implicative, since $a \rightarrow a^{2}=a \rightarrow 0=c \notin\{1\}$.

Given a filter $F$ of $L$ and $a \in L$, the congruence class of $a$ in $L / F$ will sometimes be denoted by $a_{F}$.

Proposition 19. The following conditions are equivalent for a filter $F$ of $L$ and $n \geq 1$.

(i) $F$ is $n$-fold implicative.

(ii) $L / F$ is an $n$-fold implicative residuated lattice. 
Proof. (i) $\Rightarrow$ (ii): Let $x \in L$ and $n \geq 1$. From Proposition 18(ii) it follows that $x^{n} \rightarrow x^{2 n} \in F$, or equivalently $\left(x^{n} \rightarrow\right.$ $\left.x^{2 n}\right)_{F}=1_{F}$, or equivalently $\left(x^{n}\right)_{F} \leq\left(x^{2 n}\right)_{F}$. But $x_{F}^{2 n} \leq$ $\left(x^{n+1}\right)_{F} \leq\left(x^{n}\right)_{F}$. Hence $\left(x^{n+1}\right)_{F}=\left(x^{n}\right)_{F}$ for all $x \in L$; that is, $\left(x_{F}\right)^{n+1}=\left(x_{F}\right)^{n}$ for all $x \in L$.

(ii) $\Rightarrow$ (i): Let $x, y \in L$ such that $x^{n+1} \rightarrow y \in F$; then $\left(x^{n+1} \rightarrow y\right)_{F}=1_{F}$, or equivalently $\left(x^{n+1}\right)_{F} \rightarrow y_{F}=1_{F}$; that is, $\left(x_{F}\right)^{n+1} \rightarrow y_{F}=1_{F}$. Since $L / F$ is an $n$-fold implicative residuated lattice, we have $\left(x_{F}\right)^{n} \rightarrow y_{F}=1_{F}$; thus $\left(x^{n} \rightarrow\right.$ $y)_{F}=1_{F}$, and $x^{n} \rightarrow y \in F$.

From Propositions 19 and 18, we have the following.

Proposition 20. The following conditions are equivalent for a residuated lattice $L$.

(i) L is n-fold implicative.

(ii) $\{1\}$ is an $n$-fold implicative filter of $L$.

(iii) Every filter of $L$ is $n$-fold implicative.

(iv) $x^{n}=x^{2 n}$ for all $x \in L$.

Corollary 21. Any 1-fold implicative residuated lattice is a Heyting lattice, and any Heyting lattice is an $n$-fold implicative residuated lattice.

Proof. Assume that $L$ is a 1 -fold implicative residuated lattice. If $a, b \in L$, we have $a \otimes b \leq a \wedge b=(a \wedge b) \otimes(a \wedge b) \leq a \otimes b$. Hence $a \wedge b=a \otimes b$.

An $n$-fold implicative residuated lattice may not be a Heyting lattice, as illustrated by Example 6, for $n \geq 2$.

\section{4. $n$-Fold Boolean Filter}

Definition 22. A filter of $L$ is said to be $n$-fold Boolean if $x \vee$ $\overline{x^{n}} \in F$ for all $x \in L$. [7]

In particular, any 1-fold Boolean filter is a Boolean filter.

From this definition, the class of $n$-fold boolean filters is closed under extension. Moreover, in Example $4, F_{3}=$ $\{a, b, c, 1\}$ is an $n$-fold boolean filter and $F_{2}=\{c, 1\}$ is not.

Proposition 23. The following conditions are equivalent for any filter $F$ and any $n \geq 1$.

(i) $F$ is $n$-fold Boolean.

(ii) For all $x \in L, \overline{x^{n}} \rightarrow x \in F$ implies $x \in F$.

(iii) For all $x, y \in L,\left(x^{n} \rightarrow y\right) \rightarrow x \in F$ implies $x \in F$.

Proof. (i) $\Rightarrow$ (ii): Suppose that $F$ is $n$-fold Boolean. Let $x \in L$ be such that $\overline{x^{n}} \rightarrow x \in F$; we must show that $x \in F$. By Proposition 1(12), we have $\left(x \vee \overline{x^{n}}\right) \rightarrow x=(x \rightarrow x) \wedge\left(\overline{x^{n}} \rightarrow\right.$ $x)=\overline{x^{n}} \rightarrow x \in F$; since $x \vee \overline{x^{n}} \in F$, we have $x \in F$, as $F$ is a filter.

(ii) $\Rightarrow$ (i): Let $x \in L$; we must show that $t=x \vee \overline{x^{n}} \in F$. Since $x \leq t$, we have $x^{n} \leq t^{n}$ and then $\overline{t^{n}} \leq \overline{x^{n}} \leq \overline{x^{n}} \vee x=t$. So $\overline{t^{n}} \rightarrow t=1 \in F$; by (ii), we get that $t \in F$. (ii) $\Rightarrow$ (iii): Let $x, y \in L$ such that $\left(x^{n} \rightarrow y\right) \rightarrow x \in F$; we must show that $x \in F$. By Proposition 1, we have $x^{n} \rightarrow$ $y \geq x^{n} \rightarrow 0=\overline{x^{n}}$, and then $\left(x^{n} \rightarrow y\right) \rightarrow x \leq \overline{x^{n}} \rightarrow x$. Since $\left(x^{n} \rightarrow y\right) \rightarrow x \in F$, we have $\overline{x^{n}} \rightarrow x \in F$ and $x \in F$ by (ii). (iii).

(iii) $\Rightarrow$ (ii): This follows by setting $y=0$ in condition

Remark 24. A filter $F$ satisfying Proposition 23(iii) is also said to be $n$-fold positive implicative [5].

Proposition 25. Every filter of L which is n-fold Boolean is also n-fold implicative.

Proof. Let $F$ be an $n$-fold Boolean filter of $L$ and $x, y \in L$ such that $x^{n+1} \rightarrow y \in F$. We have $\left(\overline{x^{n}} \vee x\right) \in F$. By Proposition 1, we have $\left(\overline{x^{n}} \vee x\right) \rightarrow\left(x^{n} \rightarrow y\right)=\left(\overline{x^{n}} \rightarrow\left(x^{n} \rightarrow y\right)\right) \wedge$ $\left(x^{n+1} \rightarrow y\right)=x^{n+1} \rightarrow y \in F$, since $\overline{x^{n}} \rightarrow\left(x^{n} \rightarrow y\right)=1 \epsilon$ $F$, so $x^{n} \rightarrow y \in F$, and $F$ is $n$-fold implicative.

The converse of Proposition 25 is not true, as Example 5 shows. Indeed, for $n \geq 1, F_{1}=\{1\}$ is an $n$-fold implicative filter which is not $n$-fold Boolean because $b \vee \overline{b^{n}}=b \vee a=c \notin$ $F_{1}$.

Definition 26. A residuated lattice $L$ is called $n$-fold Boolean (or $n$-fold positive implicative) if $\overline{x^{n}} \vee x=1$ for each $x \in L$.

Remark 27. Let $L$ be a residuated lattice.

(i) Since $\left(x \vee \overline{x^{n}}\right) \leq\left(x \vee \overline{x^{n+1}}\right)$, it is clear that every $n$ fold Boolean filter is $(n+1)$-fold Boolean, and $n$-fold Boolean residuated lattices are $(n+1)$-fold Boolean.

(ii) It is easy to check that the residuated lattice of Example 6 is $n$-fold boolean for $n \geq 2$. But the residuated lattice of Example 5 is not, since $a \vee \overline{a^{n}}=$ $c \neq 1$.

As in the case of $n$-fold implicativeness, one easily verifies that for a filter $F$ of a residuated lattice $L, F$ is $n$-fold boolean if and only if $L / F$ is an $n$-fold boolean residuated lattice.

Proposition 28. The following conditions are equivalent for a residuated lattice $L$.

(i) L is n-fold Boolean.

(ii) Every filter $F$ of $L$ is $n$-fold Boolean.

(iii) $\{1\}$ is an $n$-fold Boolean filter.

So, $n$-fold Boolean residuated lattices are $n$-fold implicative residuated lattices. But the converse is not true.

To end this section, we note that any 1-fold Boolean residuated lattice is a Boolean lattice, and any Boolean lattice is an $n$-fold Boolean residuated lattice, for $n \geq 1$.

However, for the residuated lattice of Example 6 and $n \geq$ 2, $L$ and $L^{E}$ are $n$-fold Boolean residuated lattices which are not Boolean lattices since $a \vee \bar{a}=c \neq 1$ (here, $E$ is any nonempty set). Also, $L$ and $L^{E}$ are not BL-algebras. 


\section{5. $n$-Fold Normal Filter}

Definition 29. A filter $F$ is $n$-fold normal if, for all $x, y \in L$, $\left(y^{n} \rightarrow x\right) \rightarrow x \in F$ implies $(x \rightarrow y) \rightarrow y \in F$.

Example 30. Let $n \geq 1$. In Example 5, simple computations prove that $F_{3}=\{1, a, c\}$ is an $n$-fold normal filter, but the filter $F_{1}=\{1\}$ is not $n$-fold normal, since $\left(c^{n} \rightarrow a\right) \rightarrow a=1 \epsilon$ $\{1\}$ and $((a \rightarrow c) \rightarrow c)=c \notin\{1\}$.

Definition 31. A residuated lattice $L$ is said to satisfy the weak double negation if the following conditions hold for all, $y \in L$.

(i) $\overline{\overline{x \otimes y}}=\overline{\bar{x}} \otimes \overline{\bar{y}}$.

(ii) $\overline{\overline{x \rightarrow y}}=\overline{\bar{x}} \rightarrow \overline{\bar{y}}$.

(iii) $\overline{\overline{(x \rightarrow y) \rightarrow y}}=\overline{\overline{(y \rightarrow x) \rightarrow x}}$.

Note. Any BL-algebra satisfies the weak double negation [17, Proposition 2.6(17)].

Example 32. (i) By simple computation, one can show that the lattice of Example 2 satisfies the weak double negation and is not a BL-algebra.

(ii) The lattice of Example 3 does not satisfy the weak double negation because $\overline{\overline{a \rightarrow b}}=c \neq 1=\overline{\bar{a}} \rightarrow \overline{\bar{b}}$.

It is easy to prove the following result.

Remark 33. If $L$ satisfies the weak double negation, then $(\overline{\bar{x}})^{n}=\overline{\overline{x^{n}}}$, for all $n \geq 1$.

Proposition 34. Let $L$ be a residuated lattice which satisfies the weak double negation and let $F$ be a filter of $L$. The following conditions are equivalent.

(i) $F$ is n-fold normal.

(ii) For every $x \in L$, if $\overline{\overline{x^{n}}} \in F$, then $x \in F$.

(iii) $D^{n}(\{1\}) \subseteq F$, where $D^{n}$ is the operator defined by $D^{n}(X):=\left\{x \in L: \overline{\overline{x^{n}}} \in X\right\}$.

Proof. (i) $\Rightarrow$ (ii): Suppose that $F$ is $n$-fold normal, and let $x \in L$ such that $\overline{\overline{x^{n}}} \in F$. Then $\left(x^{n} \rightarrow 0\right) \rightarrow 0 \in F$, and since $F$ is $n$-fold normal, then $(0 \rightarrow x) \rightarrow x \in F$; that is, $x \in F$.

(ii) $\Rightarrow$ (i): Suppose that (ii) holds, and let $\left(x^{n} \rightarrow y\right) \rightarrow$ $y \in F$. Since $\left(x^{n} \rightarrow y\right) \rightarrow y \leq(x \rightarrow y) \rightarrow y$, we have $(x \rightarrow y) \rightarrow y \in F$ and $\overline{\overline{(x \rightarrow y) \rightarrow y}} \in F$. Thus, $\overline{\overline{(y \rightarrow x) \rightarrow x}} \in F$, since $L$ satisfies the weak double negation. From this and the fact that $F$ is a filter, we have $\overline{\overline{((y \rightarrow x) \rightarrow x)^{n}}} \in F$. Thus we obtain that $((y \rightarrow x) \rightarrow$ $x) \in F$.

(ii) $\Rightarrow$ (iii): Suppose that (ii) holds, and let $x \in D^{n}(\{1\})$, that is, $\overline{\overline{x^{n}}}=1$, then $\overline{\overline{x^{n}}} \in F$, and by (ii), $x \in F$. Thus $D^{n}(\{1\}) \subseteq$ $F$ as needed.

(iii) $\Rightarrow$ (ii): Suppose $D^{n}(\{1\}) \subseteq F$, and let $x \in L$ such that $\overline{\overline{x^{n}}} \in F$. Since $L$ satisfies the weak double negation, we have $\overline{\left.\overline{\overline{\bar{x}^{n}}} \rightarrow x\right]^{n}}=\left(\overline{\overline{x^{n}}} \rightarrow \overline{\bar{x}}\right)^{n}=1^{n}=1$, so $\overline{\overline{x^{n}}} \rightarrow x \in D^{n}(\{1\}) \subseteq$ $F$. Hence, $x \in F$.

Note. (1) From the definition, it is clear that an $n$-fold normal filter is $(n+1)$-fold normal.

(2) In [18, Theorem 8], the authors state a result (in the case $n=1$ ) that was meant to be (i) $\Leftrightarrow$ (ii) above, but there seems to be a typo in the statement of their result. However, we have a correct proof, and (iii) provides an answer to their open problem. So, under the assumption of the weak double negation, the class of $n$-fold normal filters is closed under extension. lattice.

Now we give the definition of an $n$-fold normal residuated

Definition 35. $L$ is an $n$-fold normal residuated lattice if it satisfies the following condition. For all $x, y \in L, y^{n} \rightarrow x \leq x$ implies $x \rightarrow y \leq y$.

As in the case of $n$-fold implicativeness, one easily sees that, given a filter $F$ of $L, F$ is $n$-fold normal if and only if $L / F$ is an $n$-fold normal residuated lattice.

Note that the residuated lattice of Example 6 is $n$-fold normal, for $n \geq 2$.

\section{6. $n$-Fold Fantastic Filter}

Definition 36. Let $n \geq 1$. A subset $F$ of $L$ is an $n$-fold fantastic filter if $1 \in F$ and, for all $x, y \in L, y \rightarrow x \in F$ implies $\left[\left(x^{n} \rightarrow y\right) \rightarrow y\right] \rightarrow x \in F$.

In particular a 1-fold fantastic filter is a fantastic filter.

Example 37. Let $n \geq 2$.

(1) Let $L$ be the residuated lattice of Example 6. It is easy to check that $\{1\}$ is an $n$-fold fantastic filter.

(2) For the residuated lattice of Example 2. $\{1\}$ is not an $n$-fold fantastic filter since $0 \rightarrow c=1 \in\{1\}$, but $\overline{\overline{c^{n}}} \rightarrow c=\overline{\bar{c}} \rightarrow c=1 \rightarrow c=c \notin\{1\}$.

The following result gives a simple characterization of $n$ fold fantastic filters.

Proposition 38. Let $n \geq 1$ and let $F$ be a filter. $F$ is an $n$-fold fantastic filter if and only if $\left[\left(x^{n} \rightarrow y\right) \rightarrow y\right] \rightarrow(x \vee y) \in F$, for all $x, y \in L$.

Thus the class of $n$-fold fantastic filters is closed under extension.

Proof. Assume that $F$ is $n$-fold fantastic. Since $y \rightarrow(x \vee y)=$ $1 \in F$, from the hypothesis, we obtain $\left[\left((x \vee y)^{n} \rightarrow y\right) \rightarrow\right.$ $y] \rightarrow(x \vee y) \in F$.

Since $x \leq x \vee y$, we have $x^{n} \leq(x \vee y)^{n}$ and $\left[(x \vee y)^{n} \rightarrow\right.$ $y) \rightarrow y] \rightarrow(x \vee y) \leq\left[\left(x^{n} \rightarrow y\right) \rightarrow y\right] \rightarrow(x \vee y)$. Thus $\left[\left(x^{n} \rightarrow y\right) \rightarrow y\right] \rightarrow(x \vee y) \in F$.

Conversely, assume that $\left[\left(x^{n} \rightarrow y\right) \rightarrow y\right] \rightarrow(x \vee y) \epsilon$ $F$, for $x, y \in L$. 
Let $x, y \in L$ be such that $y \rightarrow x \in F$. By Proposition 1 , we have $x \vee y \leq(y \rightarrow x) \rightarrow x$, so $\left[\left(x^{n} \rightarrow y\right) \rightarrow y\right] \rightarrow$ $(x \vee y) \leq\left[\left(x^{n} \rightarrow y\right) \rightarrow y\right] \rightarrow((y \rightarrow x) \rightarrow x)$, and the latter is $(y \rightarrow x) \rightarrow\left[\left(\left(x^{n} \rightarrow y\right) \rightarrow y\right) \rightarrow x\right]$; it follows that $\left[\left(x^{n} \rightarrow y\right) \rightarrow y\right] \rightarrow x \in F$.

Proposition 39. Let $n \geq 1$. Then any $n$-fold Boolean filter is n-fold fantastic.

Proof. Assume that $F$ is $n$-fold Boolean. Let $x, y \in L$ be such that $y \rightarrow x \in F$.

We have $x \leq\left[\left(\left(x^{n} \rightarrow y\right) \rightarrow y\right) \rightarrow x\right]$, so $x^{n} \leq\left[\left(\left(x^{n} \rightarrow\right.\right.\right.$ $y) \rightarrow y) \rightarrow x]^{n}$ and $\left(x^{n} \rightarrow y\right) \geq\left[\left(\left(x^{n} \rightarrow y\right) \rightarrow y\right) \rightarrow\right.$ $x]^{n} \rightarrow y \cdot(*)$

We also have

$$
\begin{aligned}
y & \longrightarrow x \\
& \leq\left(\left(x^{n} \longrightarrow y\right) \longrightarrow y\right) \longrightarrow\left(\left(x^{n} \longrightarrow y\right) \longrightarrow x\right),
\end{aligned}
$$

by Proposition 1(15)

$$
\begin{aligned}
& =\left(x^{n} \longrightarrow y\right) \longrightarrow\left[\left(\left(x^{n} \longrightarrow y\right) \longrightarrow y\right) \rightarrow x\right] \\
& \leq\left(\left[\left(\left(x^{n} \longrightarrow y\right) \longrightarrow y\right) \longrightarrow x\right]^{n} \longrightarrow y\right) \\
& \quad \longrightarrow\left[\left(\left(x^{n} \longrightarrow y\right) \longrightarrow y\right] \longrightarrow x\right], \text { by }(*) .
\end{aligned}
$$

Since $F$ is $n$-fold positive implicative, we obtain [[( $\left(x^{n} \rightarrow\right.$ $y) \rightarrow y$ ] $\rightarrow x]] \in F$. Hence $F$ is an $n$-fold fantastic filter.

Proposition 40. Let $n \geq 1$. Any $n$-fold fantastic filter is $n$-fold normal.

Proof. Assume that $F$ is an $n$-fold fantastic filter. Let $x, y \in L$ be such that $\left(x^{n} \rightarrow y\right) \rightarrow y \in F$. We must show that $t=(y \rightarrow x) \rightarrow x \in F$.

Now, $x \leq t$ implies $\left(t^{n} \rightarrow y\right) \rightarrow y \in F$, and $y \leq t$ implies $\left(x^{n} \rightarrow y\right) \rightarrow t \in F$. Since $F$ is $n$-fold fantastic, we have $\left[\left(t^{n} \rightarrow\left(x^{n} \rightarrow y\right)\right) \rightarrow\left(x^{n} \rightarrow y\right)\right] \rightarrow t \in F(*)$.

Moreover, $x^{n} \otimes\left(t^{n} \rightarrow\left(x^{n} \rightarrow y\right)\right)=x^{n} \otimes\left(x^{n} \rightarrow\left(t^{n} \rightarrow\right.\right.$ $y)) \leq t^{n} \rightarrow y$, so by Proposition 1(14), we have $\left(t^{n} \rightarrow y\right) \rightarrow$ $y \leq\left[\left(x^{n} \otimes\left(t^{n} \rightarrow\left(x^{n} \rightarrow y\right)\right)\right] \rightarrow y=\left(t^{n} \rightarrow\left(x^{n} \rightarrow\right.\right.\right.$ $y)) \rightarrow\left(x^{n} \rightarrow y\right)$, so the latter is in $F$, and by $(*)$ we obtain $t \in F$.

Let us note that if $a, b, c \in L$, then $a \rightarrow b \leq(b \rightarrow c) \rightarrow$ $(a \rightarrow c)$; thus

$$
\begin{aligned}
(a & \longrightarrow b) \otimes(b \longrightarrow c) \\
& \leq[(b \longrightarrow c) \longrightarrow(a \longrightarrow c)] \otimes(b \longrightarrow c) \\
& \leq a \longrightarrow c .
\end{aligned}
$$

Theorem 41. Let $n \geq 1$. A filter $F$ of $L$ is $n$-fold Boolean if and only if it is $n$-fold fantastic and $n$-fold implicative.

Proof. $(\Rightarrow)$ : This follows from Propositions 25 and 39 .

$(\Leftarrow)$ : Let $F$ be $n$-fold fantastic and $n$-fold implicative, and let $x, y \in L$ be such that $\left(x^{n} \rightarrow y\right) \rightarrow x \in F$. We must show that $x \in F$.
Since $F$ is $n$-fold fantastic and $\left(x^{n} \rightarrow y\right) \rightarrow x \in F$, we have $\left[\left(x^{n} \rightarrow\left(x^{n} \rightarrow y\right)\right) \rightarrow\left(x^{n} \rightarrow y\right)\right] \rightarrow x \in F$.

From the observation above, we have $\left(x^{n} \rightarrow x^{2 n}\right) \leq$ $\left(x^{2 n} \rightarrow y\right) \rightarrow\left(x^{n} \rightarrow y\right),=\left[\left(x^{n} \rightarrow\left(x^{n} \rightarrow y\right)\right] \rightarrow\right.$ $\left(x^{n} \rightarrow y\right)$. So $\left[\left(x^{n} \rightarrow x^{2 n}\right) \rightarrow x\right] \geq\left[\left[x^{n} \rightarrow\left(x^{n} \rightarrow y\right)\right] \rightarrow\right.$ $\left.\left(x^{n} \rightarrow y\right)\right] \rightarrow x$. Thus $\left(x^{n} \rightarrow x^{2 n}\right) \rightarrow x \in F$, and $x \in F$, since $F$ is $n$-fold implicative.

Definition 42. A residuated lattice $L$ is said to be $n$-fold fantastic if for all $x, y \in L, y \rightarrow x=\left[\left(x^{n} \rightarrow y\right) \rightarrow y\right] \rightarrow$ $x$.

Example 43. Let $n \geq 2$.

(1) The residuated lattice of Example 6 is $n$-fold fantastic.

(2) The residuated lattice of Example 2 is not $n$-fold fantastic, since $a \rightarrow c=1 \neq c=\left[\left(c^{n} \rightarrow a\right) \rightarrow\right.$ a] $\rightarrow c$.

Here is a characterization of $n$-fold fantastic residuated lattices.

Proposition 44. The residuated lattice $L$ is $n$-fold fantastic if and only if the inequality $\left(x^{n} \rightarrow y\right) \rightarrow y \leq(y \rightarrow x) \rightarrow$ $x$ holds, for all $x, y \in L$.

Proof. $(\Rightarrow)$ : Assume that $L$ is an $n$-fold fantastic residuated lattice. Let $x, y \in L$.

We have $\left[\left(x^{n} \rightarrow y\right) \rightarrow y\right] \rightarrow[(y \rightarrow x) \rightarrow x]=$ $(y \rightarrow x) \rightarrow\left[\left(x^{n} \rightarrow y\right) \rightarrow y\right] \rightarrow x=1$. So $\left[\left(x^{n} \rightarrow\right.\right.$ $y) \rightarrow y] \leq[(y \rightarrow x) \rightarrow x]$, by Proposition 1(1).

$(\Leftarrow)$ : Suppose conversely that the inequality $\left(x^{n} \rightarrow\right.$ $y) \rightarrow y \leq(y \rightarrow x) \rightarrow x$ holds for all $x, y \in L$. Then $(y \rightarrow x) \rightarrow\left[\left[\left(x^{n} \rightarrow y\right) \rightarrow y\right] \rightarrow x\right]=\left[\left(x^{n} \rightarrow\right.\right.$ $y) \rightarrow y] \rightarrow[(y \rightarrow x) \rightarrow x]=1$. So $y \rightarrow x \leq$ $\left(\left(x^{n} \rightarrow y\right) \rightarrow y\right) \rightarrow x$. Now, $y \leq\left(x^{n} \rightarrow y\right) \rightarrow y$ implies that $y \rightarrow x \geq\left(\left(x^{n} \rightarrow y\right) \rightarrow y\right) \rightarrow x$, and whence the equality.

Proposition 45. The following conditions are equivalent for a residuated lattice $L$.

(i) $L$ is $n$-fold fantastic.

(ii) Every filter $F$ of $L$ is $n$-fold fantastic.

(iii) $\{1\}$ is an $n$-fold fantastic filter of $L$.

Proof. (i) $\Rightarrow$ (ii): Follows from the definitions of $n$-fold fantastic filter and $n$-fantastic residuated lattice.

(ii) $\Rightarrow$ (iii): Follows from the fact that $\{1\}$ is a filter of $L$.

(iii) $\Rightarrow(\mathrm{i})$ : Assume that $\{1\}$ is $n$-fold fantastic. Let $x, y \in$ $L$ and $t=(y \rightarrow x) \rightarrow x$. By Proposition $1, y \leq t$. So $y \rightarrow$ $t=1$ and by the hypothesis, we have $\left[\left(t^{n} \rightarrow y\right) \rightarrow y\right] \rightarrow$ $t=1$; that is, $\left[\left(t^{n} \rightarrow y\right) \rightarrow y\right] \leq t .(*)$

On the other hand, $x \leq t$ implies $x^{n} \leq t^{n}$, and hence $\left[\left(x^{n} \rightarrow y\right) \rightarrow y\right] \leq\left(t^{n} \rightarrow y\right) \rightarrow y$. Then, it follows from $(*)$ that $\left[\left(x^{n} \rightarrow y\right) \rightarrow y\right] \leq t=(y \rightarrow x) \rightarrow x$. Hence by Proposition $44, L$ is $n$-fold fantastic.

So, a filter $F$ of $L$ is $n$-fold fantastic if and only if $L / F$ is an $n$-fold fantastic residuated lattice. 
Combining Propositions 45, 28, and 20 and Theorem 41, we have the following result.

Corollary 46. Let $n \geq 1$. A residuated lattice $L$ is $n$ fold Boolean if and only if it is n-fold fantastic and $n$-fold implicative.

To end this section, we note that:

(i) $n$-fold fantastic residuated lattices are $n$-fold normal;

(ii) 1-fold fantastic residuated lattices are $M V$-algebras, and any $M V$-algebra is an $n$-fold fantastic residuated lattice, for $n \geq 1$;

(iii) however, an $n$-fold fantastic residuated lattice may not be a $B L$-algebra, as illustrated by Example 6 for $n \geq 2$.

\section{7. $n$-Fold Obstinate Filter}

Definition 47. Let $n \geq 1$. A proper filter $F$ of $L$ is said to be $n$ fold obstinate if for all $x, y \in L, x, y \notin F$ implies $x^{n} \rightarrow y \in F$ and $y^{n} \rightarrow x \in F$.

In particular a 1-fold obstinate filter is an obstinate filter.

The following result gives a characterization of $n$-fold obstinate filter.

Lemma 48. Let $n \geq 1$; a proper filter $F$ is $n$-fold obstinate if and only if, for all $x \in L, x \notin F$ implies $\overline{x^{n}} \in F$.

Proof. $(\Rightarrow)$ : Let $x \in L$ with $x \notin F$. By setting $y=0$ in the definition, we have $x^{n} \rightarrow 0 \in F$; that is, $\overline{x^{n}} \in F$.

$(\Leftarrow)$ : Conversely, let $x, y \notin F$; then $\overline{x^{n}}, \overline{y^{n}} \in F$. But $\overline{x^{n}}=$ $x^{n} \rightarrow 0 \leq x^{n} \rightarrow y$, so, $x^{n} \rightarrow y \in F$. Similarly, $y^{n} \rightarrow x \in$ F.

Remark 49. (i) An $n$-fold obstinate filter is also called an $n$ fold Boolean filter of the second kind.

(ii) Since $\overline{x^{n}} \leq \overline{x^{n+1}}$, any $n$-fold obstinate filter is $(n+1)$ fold obstinate.

Example 50. Let $L$ be the lattice of Example 2 and $n \geq 1$.

(i) The filter $F=\{b, c, d, 1\}$ is $n$-fold obstinate.

(ii) The filter $F_{2}=\{c, d, 1\}$ is not $n$-fold obstinate, since $a \notin F_{2}$ and $\overline{a^{n}}=b \notin F$.

Proposition 51. The following conditions are equivalent for any proper filter $F$ and any $n \geq 1$.

(i) $F$ is $n$-fold obstinate.

(ii) $F$ is maximal and $n$-fold Boolean.

(iii) $F$ is maximal and $n$-fold implicative.

(iv) $F$ is prime of the second kind and $n$-fold Boolean.

Proof. (i) $\Rightarrow$ (ii): Assume that $F$ is an $n$-fold obstinate filter. We first show that $F$ is maximal. Let $x \notin F$; since $F$ is $n$-fold obstinate, by Lemma $48, \overline{x^{n}} \in F$. Let $x \in L$ such that $\overline{x^{n}} \in F$; since $0 \notin F$, it is clear that $x^{n} \notin F$ and then $x \notin F$. From this, we get that, for all $x \in L, x \notin F$ if and only if $\overline{x^{n}} \in F$; hence by Proposition $9, F$ is a maximal filter.

On the other hand, let $x \in L$ such that $\overline{x^{n}} \rightarrow x \in F$. If $x \notin$ $F$, then $\overline{x^{n}} \notin F$, a contradiction, since $F$ is $n$-fold obstinate. Thus $x \in F$.

(ii) $\Rightarrow$ (iii): Follows from Proposition 25 .

(iii) $\Rightarrow$ (i): Assume that $F$ is a maximal and $n$-fold implicative filter of $L$. Let $x, y \in L$ be such that $x, y \notin F$. By Lemma 17, $F_{x}=\left\{b \in L: x^{n} \rightarrow b \in F\right\}$ is a filter of $L$ and so is $F_{y}=\left\{b \in L: y^{n} \rightarrow b \in F\right\}$.

Let $z \in F$; since $z \leq x^{n} \rightarrow z$, we have $x^{n} \rightarrow z \in F$, and hence $z \in F_{x}$ and we obtain $F \subseteq F_{x}$. On the other hand, $x^{n} \rightarrow x=1 \in F$, since $x^{n} \leq x$, and hence $x \in F_{x}$. By hypothesis, $x \notin F$. So $F \subseteq F_{x} \subseteq L$. Since $F$ is a maximal filter of $L$, we get $F_{x}=L$. Therefore, $y \in F_{x}$, or equivalently $x^{n} \rightarrow y \in F$. Similarly, we get $y^{n} \rightarrow x \in F$. Hence $F$ is an $n$-fold obstinate filter of $L$.

(ii) $\Rightarrow$ (iv): Follows from Lemma 13.

(iv) $\Rightarrow$ (i): Assume that $F$ is a prime filter of the second kind and is $n$-fold Boolean. Let $x \in L$ be such that $x \notin F$. Since $F$ is $n$-fold boolean, we have $x \vee \overline{x^{n}} \in F$. Since $F$ is a prime filter of the second kind and $x \notin F$, we have $\overline{x^{n}} \in F$. Hence $F$ is an $n$-fold obstinate filter.

We note that this is an improvement of [11, Theorem 4.14].

Definition 52. A residuated lattice $L$ is said to be $n$-fold obstinate (or $n$-fold Boolean of the second kind) if, for all $x \neq 1, x^{n}=0$.

This means that $(L \backslash\{1\} ; \otimes)$ is an $n$-nilpotent commutative semigroup.

Proposition 53. The following conditions are equivalent for any proper filter $F$ and $n \geq 1$.

(i) $L / F$ is an $n$-fold obstinate residuated lattice.

(ii) $F$ is an $n$-fold obstinate filter of $L$.

Proof. (i) $\Rightarrow$ (ii): Assume that $L / F$ is an $n$-fold obstinate residuated lattice. Let $x \in L$ be such that $x \notin F$; then, $x_{F} \neq 1_{F}$. Since $L / F$ is an $n$-fold obstinate residuated lattice, it follows that $\left(x_{F}\right)^{n}=0_{F}$. This implies $\overline{x^{n}} \in F$.

(ii) $\Rightarrow$ (i): Assume that $F$ is an $n$-fold obstinate filter of $L$. Let $x \in L$ be such that $x_{F} \neq 1_{F}$; then, $x \notin F$. It follows that $\overline{x^{n}} \in F$, or equivalently $\left(\overline{x^{n}}\right)_{F}=1_{F}$, or equivalently $\left(x^{n}\right)_{F}=$ $0_{F}$; that is, $\left(x_{F}\right)^{n}=0_{F}$.

Since $L /\{1\} \cong L$, from Proposition 53, we have the following result.

Proposition 54. Let $n \geq 1$. Then $L$ is an $n$-fold obstinate residuated lattice if and only if $\{1\}$ is an $n$-fold obstinate filter of $L$.

Thus, an $n$-fold obstinate residuated lattice is locally finite.

Example 55. Let $n \geq 2$.

(i) The lattice of Example 6 is $n$-fold obstinate. 
(ii) The lattice of Example 3 is not $n$-fold obstinate, since $d \neq 1$ and $d^{n} \neq 0$.

\section{8. $n$-Fold Integral Filter}

Definition 56. Let $F$ be a filter of the residuated lattice $L$, and $n \geq 1$.

(i) $F$ is said to be $n$-fold integral if, for all $x, y \in L$, $\overline{x \otimes y} \in F$ implies $\overline{x^{n}} \in F$ or $\overline{y^{n}} \in F$. In particular, 1 -fold integral filters are integral filters.

(ii) $L$ is said to be $n$-fold integral if, for all $x, y \in L, x \otimes$ $y=0$ implies $x^{n}=0$ or $y^{n}=0$. In particular, a 1fold integral residuated lattice is an integral residuated lattice.

\section{Example 57. Let $n \geq 2$.}

(i) In Example 5, the filter $F_{3}=\{a, c, 1\}$ is $n$-fold integral, but the filter $F_{2}=\{c, 1\}$ is not, because $\overline{a \otimes b}=1$, whereas $\overline{a^{n}}=b$ and $\overline{b^{n}}=a$.

(ii) The residuated lattice of Example 4 is $n$-fold integral, but that of Example 5 is not, since $a \otimes b=0$ and $a^{n}=$ $a \neq 0 \neq b=b^{n}$.

Proposition 58. Let $F$ be a filter of L. The following conditions are equivalent.

(i) $F$ is an $n$-fold integral filter.

(ii) $L / F$ is an $n$-fold integral residuated lattice.

Proof. (i) $\Rightarrow$ (ii): Assume that $F$ is an $n$-fold integral filter. Then, let $x, y \in L$ be such that $x_{F} \otimes y_{F}=(x \otimes y)_{F}=0_{F}$, or equivalently $\overline{(x \otimes y)_{F}}=1_{F}$; then $\overline{x \otimes y} \in F$. Since $F$ is $n$-fold integral, it follows that $\overline{x^{n}} \in F$ or $\overline{y^{n}} \in F$, so $\overline{\left(x_{F}\right)^{n}}=1_{F}$ or $\overline{\left(y_{F}\right)^{n}}=1_{F}$. From this we have $\left(x_{F}\right)^{n}=0_{F}$ or $\left(y_{F}\right)^{n}=0_{F}$.

(ii) $\Rightarrow$ (i): Let $x, y \in L$ be such that $\overline{x \otimes y} \in F$. We have $\overline{(x \otimes y)}_{F}=1_{F}$, or equivalently $(x \otimes y)_{F}=x_{F} \otimes y_{F}=0_{F}$. Since $L / F$ is an $n$-fold integral residuated lattice, we have $\left(x_{F}\right)^{n}=0_{F}$ or $\left(y_{F}\right)^{n}=0_{F}$; that is, $\overline{x^{n}} \in F$ or $\overline{y^{n}} \in F$.

From Proposition 58 and the fact that $L /\{1\} \cong L$, it is clear that the residuated lattice $L$ is $n$-fold integral if and only if $\{1\}$ is an $n$-fold integral filter.

Also, any $n$-fold integral filter is a primary filter. But the converse is not true: in Example 3, simple computations prove that $F=\{1, d\}$ is a primary filter, but not a 1 -fold integral filter, because $\overline{a \otimes a} \in F$ and $\bar{a}=c \notin F$.

Proposition 59. Any proper filter of L which is n-fold obstinate is also $n$-fold integral.

Proof. Assume that $F$ is a proper $n$-fold obstinate filter and let $x, y \in L$ such that $\overline{x \otimes y} \in F$. Since $F$ is a proper filter, it follows that $x \otimes y \notin F$, so $x \notin F$ or $y \notin F$. Since $F$ is a proper $n$-fold obstinate filter, this implies $\overline{x^{n}} \in F$ or $\overline{y^{n}} \in F$, and hence $F$ is an $n$-fold integral filter of $L$.
The converse of the above proposition is not true, since in Example $4, F_{2}=\{c, 1\}$ is an $n$-fold integral filter which is not $n$-fold obstinate because $a \notin F_{2}$ and $\overline{a^{n}}=0 \notin F_{2}$.

It follows that any $n$-fold obstinate residuated lattice is $n$ fold integral.

Definition 60. A filter $F$ satisfies $n$-fold double negation if, for all $x \in L, \overline{\overline{x^{n}}} \rightarrow x \in F$ implies $\overline{\left((\bar{x})^{n}\right)} \rightarrow x \in F$.

Example 61. Let $n \geq 2$.

(i) In Example 2, we see by simple computations that the filter $F_{2}=\{c, d, 1\}$ satisfies $n$-fold double negation.

(ii) In Example 3, the filter $F=\{1, d\}$ does not satisfy $n$ fold double negation since $\overline{\overline{a^{n}}} \rightarrow a=1 \in F$ but $\overline{\left((\bar{a})^{n}\right)} \rightarrow a=a \notin F$.

Proposition 62. Let $F$ be a proper filter which satisfies $n$-fold double negation. If $F$ is $n$-fold integral and $n$-fold fantastic, then $F$ is n-fold obstinate.

Proof. Assume that $F$ is $n$-fold integral and $n$-fold fantastic. Let $x \in L$ such that $x \notin F$. By Proposition 1 , we have $\overline{(x \otimes \bar{x})}=$ $1 \in F$. Since $F$ is $n$-fold integral, we have $\overline{x^{n}} \in F$ or $\overline{(\bar{x})^{n}} \in F$.

Now, by Proposition 38, $\overline{\overline{x^{n}}} \rightarrow x \in F$ as $F$ is $n$-fold fantastic; thus $\overline{(\bar{x})^{n}} \rightarrow x \in F$ by $n$-fold double negation. Since $x \notin F$, we also have $\overline{(\bar{x})^{n}} \notin F$, so $\overline{x^{n}} \in F$.

Definition 63. A residuated lattice $L$ satisfies $n$-fold double negation if, for all $x \in L, \overline{\overline{x^{n}}} \rightarrow x=1$ implies $\overline{\left((\bar{x})^{n}\right)} \rightarrow$ $x=1$.

One easily verifies that a residuated lattice $L$ satisfies $n$ fold double negation if and only if so does filter $\{1\}$.

Example 64. Let $n \geq 2$. The residuated lattice of Example 2 satisfies $n$-fold double negation. But the lattice of Example 3 does not, since $\overline{\overline{a^{n}}} \rightarrow a=1$ but $\overline{\left((\bar{a})^{n}\right)} \rightarrow a=a \neq 1$.

From Propositions 62 and 45, we obtain the following corollary.

Corollary 65. Let $L$ be a residuated lattice satisfying $n$-fold double negation. If $L$ is $n$-fold integral and $n$-fold fantastic, then $L$ is $n$-fold obstinate.

\section{9. $n$-Fold Involutive filter}

In [11], Zahiri and Farahani introduced the notion of $n$-fold involutive filter of $M T L$-algebra. In this section, we follow their idea and give the corresponding definition on residuated lattices.

Definition 66. Let $F$ be a filter of the residuated lattice $L$.

(i) $F$ is called an $n$-fold involutive filter of $L$ (or $n$-fold IRL filter) if $\overline{\overline{x^{n}}} \rightarrow x \in F$, for all $x \in L$. 
(ii) $L$ is called an $n$-fold involutive residuated lattice (or $n$-fold IRL) if $\overline{\overline{x^{n}}} \rightarrow x=1$, for all $x \in L$.

Remark 67. (i) It is easy to verify that $F$ is an $n$-fold involutive filter if and only if $L / F$ is an $n$-fold involutive residuated lattice.

(ii) The class of $n$-fold involutive filters is closed under extension.

These two notions are weakenings of the corresponding ones for $n$-fold fantastic, by setting $y=0$ in Definitions 36 and 42 . So, $n$-fold fantastic filters are $n$-fold involutive

In [11], they also study the notion of extended involutive filter of $M T L$-algebra, by taking $x=0$ in Definition 29 .

Definition 68. (i) $F$ is called an $n$-fold extended involutive filter of the residuated lattice $L$ (or $n$-fold EIRL filter) if $\overline{\overline{x^{n}}} \in$ $F$ implies $x \in F$, for all $x \in L$.

(ii) $L$ is called an $n$-fold extended involutive residuated lattice (or $n$-fold EIRL) if $\overline{\overline{x^{n}}}=1$ implies $x=1$, for all $x \in L$.

Clearly, $F$ is an $n$-fold extended involutive filter if and only if $L / F$ is an $n$-fold extended involutive residuated lattice. Moreover, an $n$-fold involutive filter (an $n$-fold normal filter, resp.) is $n$-fold extended involutive.

Now, let us consider some statements about $F$.

$$
\begin{aligned}
& \left(S_{1}\right) \forall x \in L, \overline{(\bar{x})^{n}} \rightarrow \overline{\overline{x^{n}}} \in F . \\
& \left(S_{2}\right) \forall x \in L, \overline{(\bar{x})^{n}} \rightarrow x \in F . \\
& \left(S_{3}\right) \forall x \in L, \overline{\overline{x^{n}}} \rightarrow x \in F \quad(n \text {-fold involutive filter). } \\
& \left(S_{4}\right) \forall x \in L, \overline{\overline{x^{n}}} \rightarrow x \in F \text { implies } \overline{(\bar{x})^{n}} \rightarrow x \in \\
& F \quad(n \text {-fold double negation). }
\end{aligned}
$$

Then we have the following implications.

$\left(S_{2}\right)$ implies $\left(S_{3}\right)$, because $\overline{\overline{x^{n}}} \leq \overline{(\bar{x})^{n}}$. Moreover, by repeated use of Proposition 1 (14) and (15), we have $\left(S_{1}\right) \Rightarrow$ $\left(S_{4}\right) ;\left(S_{1}\right)$ and $\left(S_{3}\right) \Rightarrow\left(S_{2}\right) ;\left(S_{3}\right)$ and $\left(S_{4}\right) \Rightarrow\left(S_{2}\right)$. So, $\left(S_{3}\right)$ and $\left(S_{4}\right) \Rightarrow\left(S_{2}\right) \Rightarrow\left(S_{3}\right)$. Then, we easily obtain the following result.

Proposition 69. Let $F$ be a filter of the residuated lattice $L$

(i) If $F$ satisfies $n$-fold double negation, then $F$ is $n$-fold involutive if and only if $F$ satisfies $\left(S_{2}\right)$.

(ii) If $F$ is $n$-fold involutive, then $F$ satisfies $n$-fold double negation if and only if $F$ satisfies $\left(S_{2}\right)$.

Now, it is easy to see that an $n$-fold Boolean filter satisfies $n$-fold double negation if and only if it satisfies $\left(S_{2}\right)$. So it needs not to satisfy any one of them, as can be observed on filter $\{1\}$ of Example 6. follows.

We may restate [11, Theorem 3.14, Theorem 3.16] as

Theorem 70. Let $F$ be a filter of the residuated lattice $L$.

(i) If $F$ is $n$-fold Boolean, then $F$ is $n$-fold implicative and $n$-fold extended involutive. The converse holds if $F$ satisfies $n$-fold double negation. (ii) If L is n-fold Boolean, then L is n-fold implicative and nfold extended involutive. The converse holds if L satisfies n-fold double negation.

Now, here are some consequences of $\left(S_{1}\right)$.

Theorem 71. Let $F$ be a filter satisfying $n$-fold double negation $\left(\operatorname{or}\left(S_{1}\right)\right)$. Then $F$ is $n$-fold obstinate if and only if $F$ is $n$-fold integral and $n$-fold involutive.

Proof. This follows from Propositions 39, 51, 59, and 69 and the proof of Proposition 62.

Theorem 72. Let $F$ be a filter of L satisfying $\left(S_{1}\right)$. Then $F$ is $n$-fold obstinate if and only if $F$ is $n$-fold integral and $n$-fold extended involutive.

Proof. $(\Rightarrow)$ : This follows from Theorem 71 , the fact that $\left(S_{1}\right)$ implies $\left(S_{4}\right)$, and the fact that an $n$-fold involutive filter is $n$ fold extended involutive.

$(\Leftarrow)$ : Let $x \in L$ such that $x \notin F$. Since $\overline{x \otimes \bar{x}}=1 \in F$, we have $\overline{x^{n}} \in F$ or $\overline{(\bar{x})^{n}} \in F$.

If $\overline{(\bar{x})^{n}} \in F$, then by $\left(S_{1}\right)$ we would have $\overline{\overline{x^{n}}} \in F$, and $x \in F$ as $F$ is $n$-fold EIRL; a contradiction. So, $\overline{(\bar{x})^{n}} \notin F$, and $\overline{x^{n}} \in$ F.

\section{Conflict of Interests}

The authors declare that there is no conflict of interests regarding the publication of this paper.

\section{Acknowledgment}

The authors wish to thank the referees for their excellent suggestions that improved the presentation and the readability of the paper.

\section{References}

[1] P. Hájek, Metamathematics of Fuzzy Logic, vol. 4 of Trends in Logic, Studia Logica Library, Kluwer Academic, Dordrecht, The Netherlands, 1998.

[2] M. Haveshki and E. Eslami, " $n$-fold filters in BL-algebras," $M L Q$. Mathematical Logic Quarterly, vol. 54, no. 2, pp. 176-186, 2008.

[3] C. Lele, "Algorithms and computations in BL-algebra," International Journal of Artificial Life Research, vol. 1, no. 4, pp. 29-47, 2010.

[4] S. Motamed and A. B. Saeid, "n-Fold obstinate filters in BLalgebras," Neural Computing and Applications, vol. 20, no. 4, pp. 461-472, 2011.

[5] E. Turunen, N. Tchikapa, and C. Lele, "A new characterization for $\mathrm{n}$-fold positive implicative BL-logics," Communications in Computer and Information Science, vol. 297, no. 1, pp. 552-560, 2012.

[6] M. Haveshki, A. B. Saeid, and E. Eslami, "Some types of filters in BL algebras," Soft Computing, vol. 10, no. 8, pp. 657-664, 2006.

[7] B. Van Gasse, G. Deschrijver, C. Cornelis, and E. E. Kerre, "Filters of residuated lattices and triangle algebras," Information Sciences, vol. 180, no. 16, pp. 3006-3020, 2010. 
[8] M. Kondo and W. A. Dudek, "Filter theory of BL algebras," Soft Computing, vol. 12, no. 5, pp. 419-423, 2008.

[9] M. Kondo and E. Turunen, "Prime filters on residuated lattices," in Proceedings of the 42nd IEEE International Symposium on Multiple-Valued Logic (ISMVL '12), pp. 89-91, May 2012.

[10] A. Borumand Saeid and M. Pourkhatoun, "Obstinate filters in residuated lattices," Bulletin Mathématique de la Société des Sciences Mathématiques de Roumanie, vol. 55(103), no. 4, pp. 413-422, 2012.

[11] O. Zahiri and H. Farahani, "n-fold filters of MTL-algebras," Afrika Matematika, 2013.

[12] M. Kondo, "Classification of residuated lattices by filters," School of Information Environment, vol. 1769, pp. 33-38, 2011.

[13] P. M. Idziak, "Lattice operation in BCK-algebras," Mathematica Japonica, vol. 29, no. 6, pp. 839-846, 1984.

[14] C. Buşneag and D. Piciu, "The stable topology for residuated lattices," Soft Computing, vol. 16, no. 10, pp. 1639-1655, 2012.

[15] S. Ghorbani and L. Torkzadeh, "Nilpotent elements of residuated lattices," International Journal of Mathematics and Mathematical Sciences, vol. 2012, Article ID 763428, 9 pages, 2012.

[16] G. Georgescu and L. Leuştean, "Some classes of pseudo-BL algebras," Journal of the Australian Mathematical Society, vol. 73, no. 1, pp. 127-153, 2002.

[17] C. Lele and J. B. Nganou, "MV-algebras derived from ideals in BL-algebras," Fuzzy Sets and Systems, vol. 218, pp. 103-113, 2013.

[18] A. Borumand Saeid and S. Motamed, "Normal filters in BLalgebras," World Applied Sciences Journal, vol. 7, pp. 70-76, 2009. 


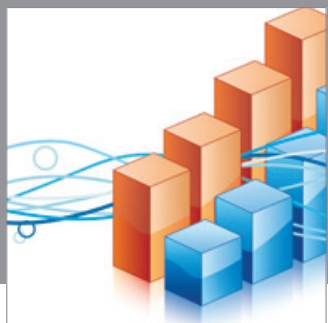

Advances in

Operations Research

mansans

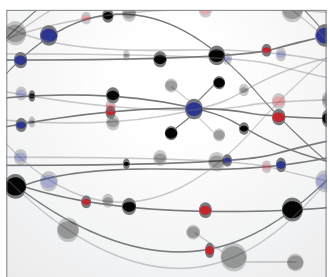

The Scientific World Journal
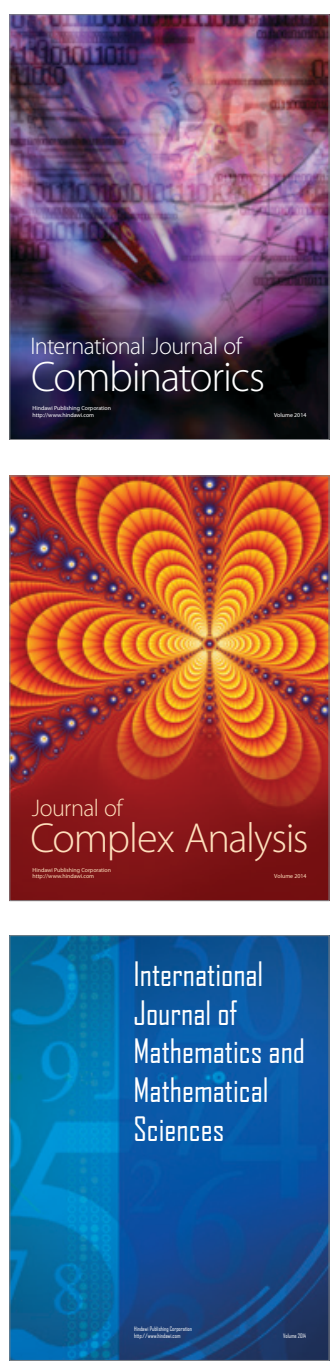
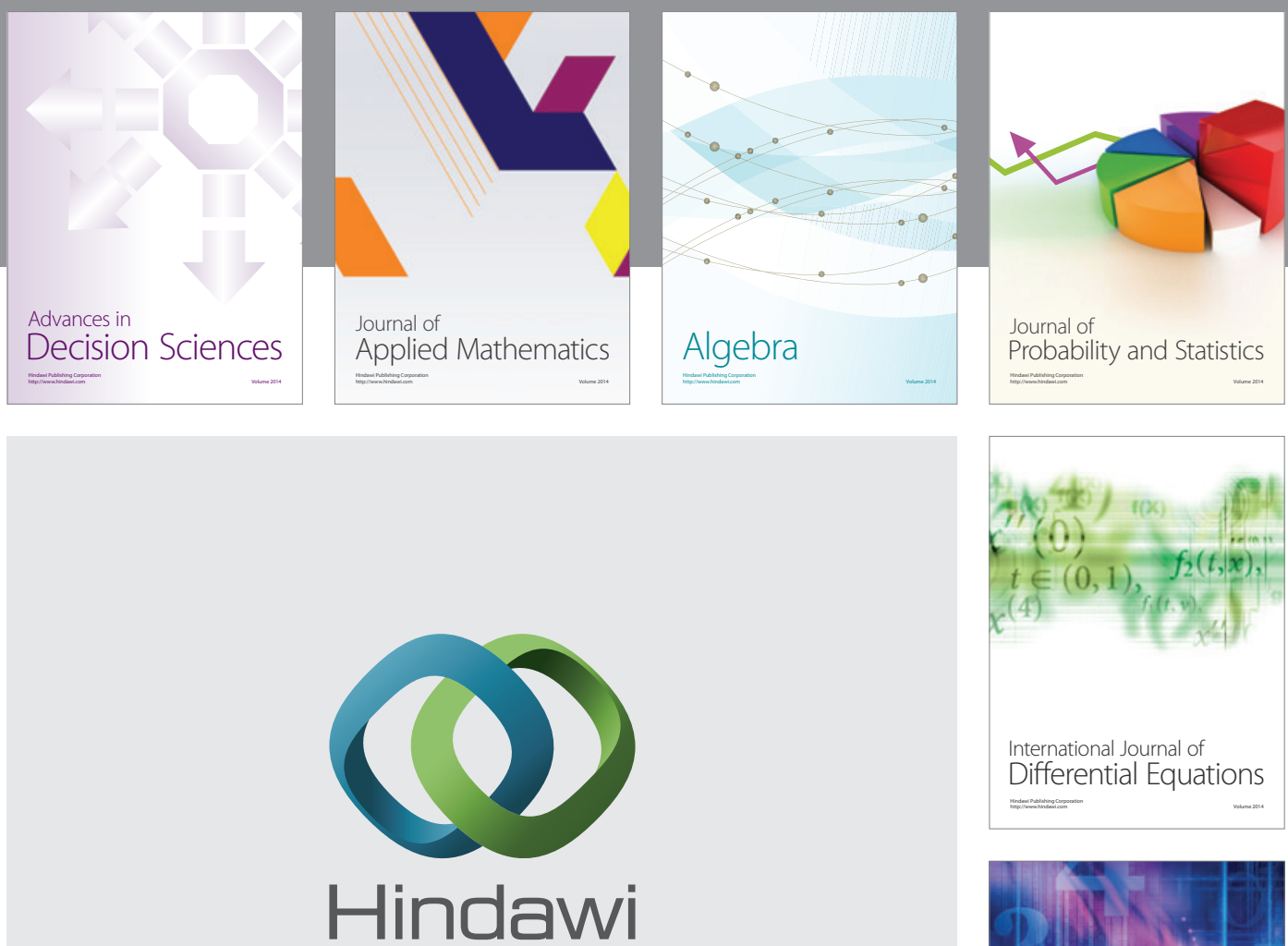

Submit your manuscripts at http://www.hindawi.com
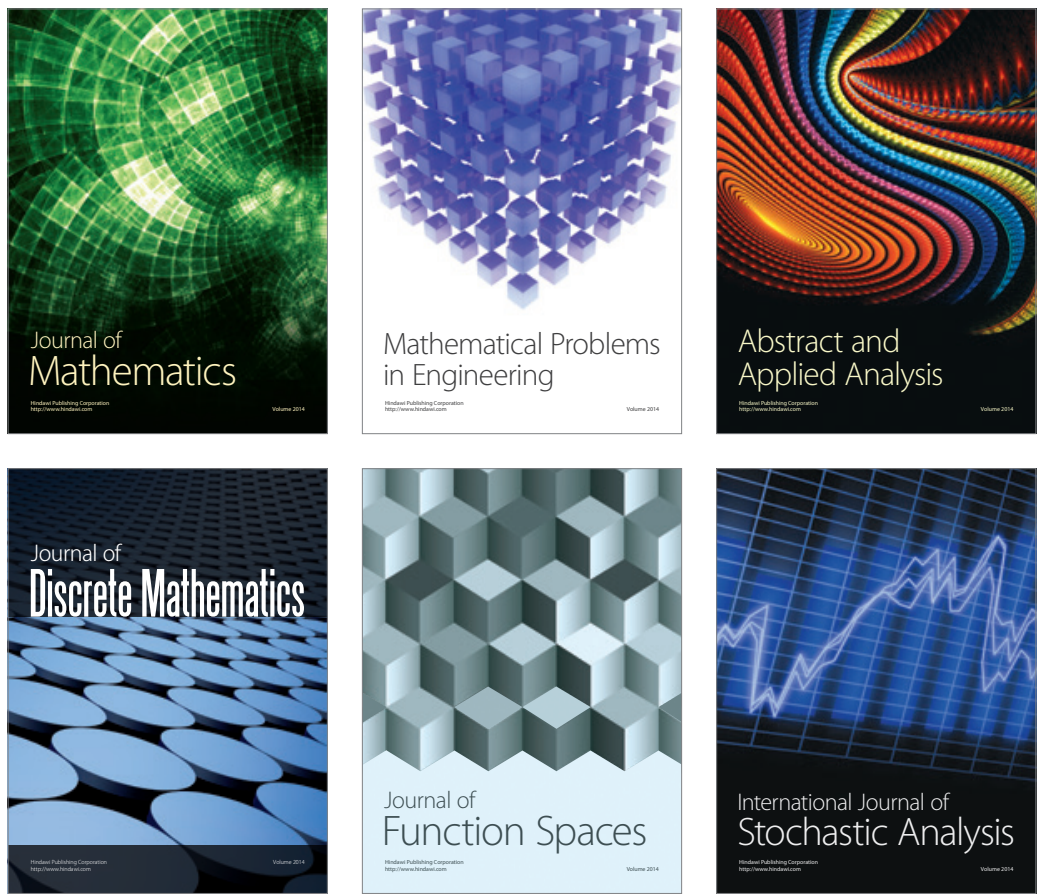

Journal of

Function Spaces

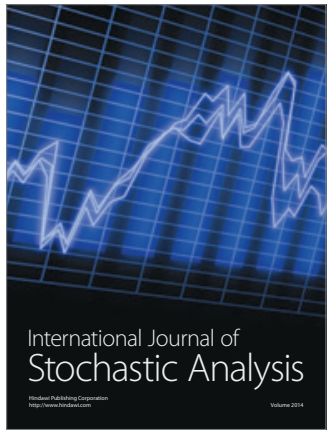

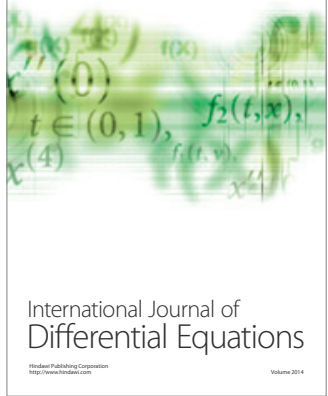
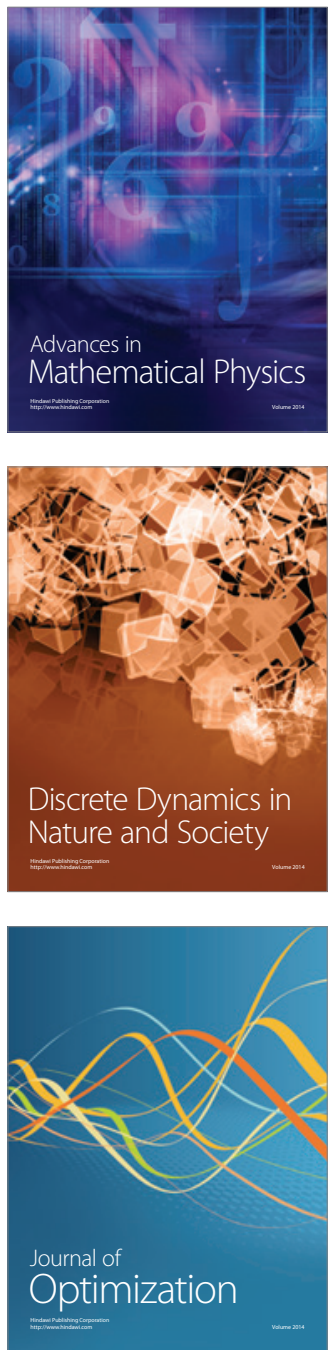